CERN-TH/99-330

hep-th/9910268

\title{
Conformal Field Theories on K3 and Three-Dimensional Gauge Theories
}

\author{
P. Mayr \\ Theory Division, CERN, 1211 Geneva 23, Switzerland
}

\begin{abstract}
According to a recent conjecture, the moduli space of the heterotic conformal field theory on a $G \subset \mathrm{ADE}$ singularity of an ALE space is equivalent to the moduli space of a pure $\mathcal{N}=4$ supersymmetric three-dimensional gauge theory with gauge group $G$. We establish this relation using geometric engineering of heterotic strings and generalize it to theories with non-trivial matter content. A similar equivalence is found between the moduli of heterotic CFT on isolated Calabi-Yau 3-fold singularities and two-dimensional Kazama-Suzuki coset theories.
\end{abstract}

October 1998 


\section{Introduction}

In a recent paper [1], the exact world-sheet instanton corrected moduli space of the heterotic conformal field theory on a $G=A_{1}$ singularity of $\mathrm{K} 3$ has been determined in the $M_{s t r} \rightarrow \infty$ limit and found to be given by the Atiyah-Hitchin manifold [2]. This is the same moduli as that of the three-dimensional pure $\mathcal{N}=4$ theory [3] with gauge group $G=A_{1}$. It has been conjectured in [1] that this relation between the moduli spaces of the conformal field theory and the $3 \mathrm{~d}$ gauge theories holds for any $G$. For $G=A_{n}$, the proposal is verified by the analysis of [4].

The three dimensional $\mathcal{N}=4$ supersymmetric gauge theories can be studied by a circle compactification of the four dimensional $\mathcal{N}=2$ theories [3]. The instantons in the three dimensional theory correspond to monopoles and dyons in four dimensions. This situation can also be studied in the field theory limit of a type II compactification on a Calabi-Yau $W$ times $S^{1}$. In this theory, the charged states of the field theory are represented by Dbrane wrappings on small $p$-cycles $C_{p}$ of a local singularity in $W$; in the limit $\alpha^{\prime} \rightarrow 0$ with $V\left(C_{p}\right) /\left(\alpha^{\prime}\right)^{p / 2}$ fixed, most of the fundamental states and gravity decouple and one is left with the light spectrum of the field theory. E.g., in the type IIB theory, the monopole in four dimensions is a D3 brane wrapped on a small 3-cycle $C_{3}$ of a singularity in a local patch of $W$. The instanton in 3d is the Euclidean wrapping of the D3 brane on $C_{3} \times S^{1}$.

The gauge coupling $g_{3}$ of the three-dimensional field gets perturbative contributions at tree level and one-loop as well as possible instanton corrections. The instanton correction is of the form $e^{-(I+i \sigma)}$, where $\sigma$ is the scalar dual to the photon and the instanton action $I$ is approximately $2 \pi M r$ for large $r$, where $M$ is the mass of the monopole in four dimensions. In the type II theory the latter is given by the volume of the brane wrapping $V\left(C_{3}\right)$.

The three dimensional gauge theories enjoy often interesting dualities that equate the Coulomb branch of a certain theory with the Higgs branch of another [5] [6] [7]. In the type II string, this duality is interpreted as a T-duality on the $S^{1}$ which takes the type IIB on $W \times S^{1}(r)$ to type IIA on $W \times S^{1}(1 / r)$. If $W$ is a singularity of small 3 -cycles as in the previous discussion, the field theory limit of type IIA describes a theory of hypermultiplets parametrizing the volumes of, and RR fields on, the cycles $C_{3}^{i}$. This is the theory that should be naturally identified with the heterotic CFT in the duality discussed in this paper. The D3 instantons of the type IIB theory become Euclidean D2 branes wrapped on $C_{3}$ after the T-duality.

In the heterotic CFT, the expansion is in terms of world-sheet instantons and the corresponding instanton action of the form $e^{-\frac{V(c)}{\alpha^{\prime}}}$, where $c$ is a 2-cycle in the K3 of the 
heterotic compactification on which a Euclidean fundamental string is wrapped. Moreover

tree-level and one-loop of the field theory correspond to the $\alpha^{\prime 0}$ and $\alpha^{\prime 1}$ contribution to the heterotic string metric, respectively [1]. To obtain the Hyper-Kähler moduli space of the field theory one takes a similar field theory limit of the heterotic string with $\alpha^{\prime} \rightarrow 0$ at fixed $V(c) / \alpha^{\prime}$. The moduli space of hypermultiplets describes the local deformations of an ADE singularity of an ALE space that governs the local patch of the K3 in this limit.

Although the expansion of the gauge theory and the CFT is morally speaking around the same point, namely in the limit of large instanton action or large volume of the 2cycles $c$ in the heterotic string or $C$ in the type IIA theory, respectively, it would be hasty to conclude that the corresponding instanton expansions are exactly the same. In fact it would be surprising if the wrappings of the D2 brane instantons of the type IIA theory would follow the same rule as that of the fundamental world-sheet instantons of the heterotic CFT. It would be interesting to relate the two instanton expansions.

In this note we derive the conjecture of ref. [1] by using heterotic/type II duality to show that the type II geometry $W$ associated to the heterotic string the ADE singularity is the appropriate, known Calabi-Yau singularity of small 3-cycles that yields the $G$ gauge theory upon type IIB compactification on $W \times S^{1}$. We will also consider various generalizations of the conjecture.

\section{Field theory limit}

Consider the heterotic string compactification on $K 3 \times T^{2}$, where the $\mathrm{K} 3$ will be replaced by the ADE singularity momentarily. In general this is dual to type IIA on a K3 fibered Calabi-Yau 3-fold $W$. If we assume that the bundle factorizes on $\mathrm{K} 3 \times T^{2}$ and the heterotic K3 is elliptically fibered, the four-dimensional duality can be pushed up to six dimensions, between F-theory on the, now elliptically fibered, Calabi-Yau $W$ and heterotic string on K3.

Let us first show that the the field theory limit of the heterotic string described by the CFT on the ADE singularity with a Hyper-Kähler moduli space corresponds to a special - field theory limit of the type IIA theory The heterotic parameters behave as

$$
g_{\text {het }} \rightarrow 0, \quad \alpha^{\prime}{ }_{h e t} \rightarrow 0, \quad V(c) / \alpha^{\prime}{ }_{h e t}=\text { const. }
$$

where $c$ stand collectively for a 2-cycle in the ADE singularity. 
First note that all $p$-cycles of K3 except for the cycles $c$ of the singularity grow as $\alpha^{\prime-p / 2}$ in the limit (2.1). This holds in particular for the two universal classes $E$ and $B$ of the elliptically fibered $\mathrm{K} 3$ with a section, where $E$ is the class of the fiber and $B$ the class of a section. Consider the singularity from a collision of singular elliptic fibers at a point $s_{0}$ of $B$. The transverse dimensions to the singularity are described by $E$ and $B$, which become non-compact in the above limit. Therefore we are left with the local singularity of a non-compact ALE space.

The four-dimensional compactification above can be considered as a compactification of the six-dimensional duality between type IIA on $\mathrm{K} 3$ and heterotic string on $T^{4}$ on a base $\mathbf{P}^{1}$ denoted by $B$. From the relations in [8] one can then easily see that the four-dimensional coupling constants are related by

$$
\frac{1}{g_{4, \text { het }}^{2}} \sim \frac{V_{\text {het }}(B)}{g_{6, \text { het }}^{2}} \sim V_{I I}(B), \quad \frac{1}{g_{4, I I}^{2}} \sim \frac{V_{I I}(B)}{g_{6, I I}^{2}} \sim V_{\text {het }}(B) .
$$

Note that $B$ is the base of the elliptically fibered K3 of the heterotic theory and the K3 fibration of $W$ in the type IIA theory, respectively. Since $V_{\text {het }}(B)$ becomes large, we have a weakly coupled type IIA theory. To have a weakly coupled heterotic string, $V_{I I}(B)$ becomes large, too.

Moreover the type IIA string is obtained from wrapping a heterotic 5-brane on $T^{2}$ times the elliptic fiber of K3. For generic volume of $T^{2}$ this 4-cycle becomes very large and therefore $\alpha^{\prime} \rightarrow 0$ in the type II theory. However as we will show in the next section, the limit we have to take is not the naive $\alpha^{\prime} \rightarrow 0$ limit: to the small 2-cycles $c_{i}$ in K3 correspond small 3-cycles $C_{i}$ in $W$ with a fixed volume $V\left(C_{i}\right) / \alpha_{I I}^{3 / 2}$.

Above we have recovered the definition of the field theory limit of the dual type II string studied in the geometric engineering approach of type II strings [9] on CalabiYau singularities. The fact that it is the fiber of the heterotic K3 $Z$ that gets large (and not just the 4-cycle of the 5-brane wrapping) implies a special limit in the complex structure of the Calabi-Yau singularity. We will show in the next section that the special singularities one obtains in this way are precisely those used in [10] to extend the geometric engineering approach of [9] to describe heterotic moduli spaces by F-theory limits of CalabiYau singularities. The subsequent sections may be understood without going through the mathematics of the next section. 


\section{Dual type IIA singularities and stable degenerations}

A second consequence of the large elliptic fiber of $Z$ is a limit in the complex structure of $W$ that is known as a stable degeneration [11] [12] [13]. In particular it was proposed in [11] that if $W$ is a $\mathrm{K} 3$ manifold, one can see the heterotic $T^{2}$ in $W$ on the nose in the limit of the stable degeneration, which in heterotic terms corresponds to a very large $T^{2}$. Since we are interested in the complex structure of $W$ parametrized by the hypermultiplets of the type IIA theory, we can consider F-theory compactification on $W$ dual to heterotic on $Z$ without the extra $T^{2}$. The type IIA theory is related to F-theory by the extra $T^{2}$ compactification which will affect only the Kähler moduli space of the Calabi-Yau $W$.

In the following we will describe in some detail general deformations of the stable degeneration and make contact with the field theory limit of the type IIA on non-compact singularities considered in [10]. We will verify the identifications made in [11] and add also the information about the bundles on the heterotic manifold $Z$ in this way. This will serve as a starting point for the identification of the type II singularity dual to the ADE singularity of the heterotic string in the next section.

The elliptically fibered manifold $W$ for F-theory compactification in Weierstrass form is given by

$$
p_{W}=y^{2}+x^{3}+x f+g=0,
$$

with $f$ and $g$ some functions depending on the coordinates on the base $B$ of the elliptic fibration $\pi: W \rightarrow B$. To describe the limit in complex structure we are interested in, we consider families of the F-theory manifold $W$ in a fibration over the complex plane, similarly as in [13]. Let us write (3.1) in more detail as

$$
p_{W}=y^{2}+x^{3}+x \sum_{\alpha} s^{4-\alpha} \tilde{s}^{4+\alpha} f_{\alpha}^{(4)}+\sum_{\alpha} s^{6-\alpha} \tilde{s}^{6+\alpha} f_{\alpha}^{(6)},
$$

where $f_{\alpha}^{(k)}$ are constants if $W$ is a K3, but more generally functions on the $n-1$ dimensional base $B^{\prime}$ of the K3 fibration $\pi^{\prime}: W \rightarrow B^{\prime}$ of the Calabi-Yau $n+1$-fold W. Moreover $s$ and $\tilde{s}$ are homogeneous coordinates on the base $\mathbf{P}^{1}$ of the elliptic fibration of the K3 fiber.

To perform the limit, we consider families $Y$ of $W$ in a fibration over another $\mathbf{P}^{1}$ parametrized by $\mu$

$$
p_{Y}=y^{2}+x^{3}+x \sum_{\alpha, \beta} s^{4-\alpha} \tilde{s}^{4+\alpha} \mu^{4-\beta} f_{\alpha}^{(4)} a_{\alpha, \beta}^{(4)}+\sum_{\alpha, \beta} s^{6-\alpha} \tilde{s}^{6+\alpha} \mu^{6-\beta} f_{\alpha}^{(6)} a_{\alpha, \beta}^{(6)},
$$


where $a_{\alpha, \beta}^{(k)}$ are some complex constants that we will set to 0 or 1 in the following. In general, the fiber of $Y$ at $\mu=0$ is a (certain deformation) of the original manifold $W$. However if we restrict the complex structure of $Y$ such that $a_{\alpha, \beta}^{(k)}=0$ for $\alpha+\beta>k$ the fiber at $\mu=0$ becomes

$$
p_{Y}=y^{2}+x^{3}+x s^{4} \sum_{\alpha \leq 0} s^{-\alpha} \tilde{s}^{4+\alpha} f_{\alpha}^{(4)}+s^{6} \sum_{\alpha \leq 0} s^{-\alpha} \tilde{s}^{6+\alpha} f_{\alpha}^{(6)} \text {, }
$$

which has a non-minimal singularity at $y=x=s=0$. After a blow up of $Y$,

$$
y=\rho^{3} y, x=\rho^{2} x, s=\rho s, \mu=\rho \mu,
$$

we arrive at

$$
p_{Y^{\prime}}=y^{2}+x^{3}+x \sum_{\alpha, \beta} s^{4-\alpha} \tilde{s}^{4+\alpha} \mu^{4-\beta} \rho^{4-\alpha-\beta} f_{\alpha}^{(4)}+\sum_{\alpha, \beta} s^{6-\alpha} \tilde{s}^{6+\alpha} \mu^{6-\beta} \rho^{6-\alpha-\beta} f_{\alpha}^{(6)} .
$$

The singular fiber at $\mu=0$ is now replaced by a reducible fiber $\hat{W}$ with two components corresponding to $\mu=0$ and $\rho=0$ :

$$
\begin{aligned}
p_{W_{1}}=p_{0}+p_{+}= & \left(y^{2}+x^{3}+x f_{0}^{(4)}+f_{0}^{(6)}\right)+ \\
& \left(x \sum_{\alpha<0} \tilde{s}^{4+\alpha} \rho^{-\alpha} f_{\alpha}^{(4)}+\sum_{\alpha<0} \tilde{s}^{6+\alpha} \rho^{-\alpha} f_{\alpha}^{(6)}\right), \\
p_{W_{2}}=p_{0}+p_{-}= & \left(y^{2}+x^{3}+x f_{0}^{(4)}+f_{0}^{(6)}\right)+ \\
& \left(x \sum_{\alpha>0} s^{4-\alpha} \mu^{\alpha} f_{\alpha}^{(4)}+\sum_{\alpha>0} s^{6-\alpha} \mu^{\alpha} f_{\alpha}^{(6)}\right) .
\end{aligned}
$$

The two components $W_{1}$ and $W_{2}$ intersect over the locus $\mu=\rho=0$ described by $p_{0}=0$. According to [11] this intersection is to be identified with the heterotic manifold $Z$.

This proposal can be made precise using local mirror symmetry which allows to also extract the heterotic bundle [10]. As shown there, a type IIA compactification on certain non-compact Calabi-Yau singularities $\mathcal{W}$ results in a gravity free field theory with the moduli space of holomorphic stable bundles on a compact Calabi-Yau $Z_{n}$ defined as a certain hypersurface in $\mathcal{W}$. For appropriate structure group of the bundle this data can be interpreted as a heterotic compactification in the point particle limit. From comparison with (3.3), one observes that if we consider separately a manifold $\mathcal{W}_{1}$ described by $p_{W_{1}}=0$, it describes precisely a non-compact Calabi-Yau singularity of the type considered in [10]. The singularity $\mathcal{W}_{1}$ indeed describes the moduli space of holomorphic stable bundles $V$ on $Z: p_{0}=0$. Moreover we can use the identifications for the singularity $\mathcal{W}_{1}$ derived in [10] to read off the moduli of the bundle $V$ on $Z$. 


\section{Chains and cycles in $\hat{W}$}

Let us show now how the vanishing 2 cycles $c_{i}$ of the heterotic ADE singularity correspond to shrinking 3 -cycles in the dual type II manifold $\hat{W}$. The latter support the D2 instantons of the field theory limit of the type IIA theory and the monopole wrappings of the T-dual type IIB theory that represents the Coulomb branch of the three-dimensional gauge theory.

Let us start with the illustrative and simpler case of F-theory on a $\mathrm{K} 3 \mathrm{~W}$ dual to heterotic string on $T^{2}$. It shares many of the relevant aspects with the case of the heterotic string on K3 that will be obtained by fibering over an extra $\mathbf{P}^{1}$.

Before the stable degeneration, $W$ is an elliptic fibration over a $\mathbf{P}^{1}$ with 24 singular elliptic fibers located at the zeros of the discriminant $\Delta=4 f^{3}+27 g^{2}$, where $f$ and $g$ are degree 8 and 12 polynomials in the variable $s$ on the base $\mathbf{P}^{1}$, respectively. After the stable degeneration, $W$ has split into the two components $W_{1}$ and $W_{2}$ described by eq. (3.3), intersecting over the elliptic curve $E: p_{0}=0$. The base of the elliptic fibrations of the elliptic rational surfaces $W_{i}$ are two $\mathbf{P}^{1}$ 's that intersect over a point, $\mu=\rho=0$. There are 12 points on each $\mathbf{P}^{1}$ above which the elliptic fibers becomes singular:
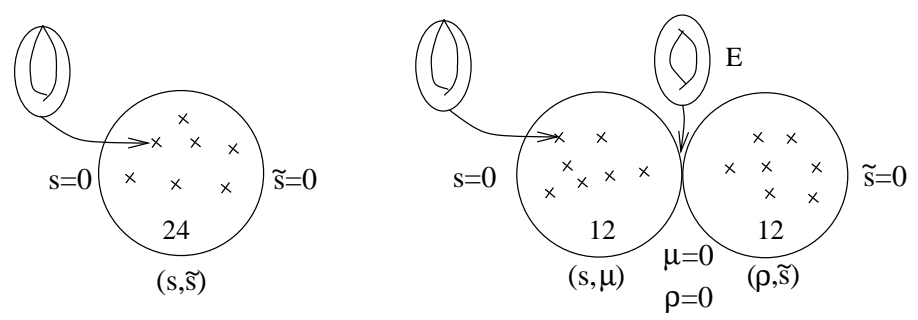

Fig. 1: The original K3 and the generic stable deformation.

There are two basic degenerations of this elliptic fibration. First we can bring together singular elliptic fibers of a single component, say $W_{1}$. This introduces a singularity of small 2-cycles in $W_{1}$ with a type determined by the classification of Kodaira. Since the relative location of the singular elliptic fibers is described by $p_{+}$in eq.(3.3), with parameters that translates to that of the heterotic bundle as described in [10], this corresponds to a degeneration of the gauge bundle in the heterotic string on $T^{2}$.

The second kind of degeneration consists of bringing together a singular fiber from $W_{1}$ with another singular fiber from $W_{2}$. This is only possible at the intersection of the two base $\mathbf{P}^{1}$ 's at $\mu=\rho=0$. Again we obtain a shrinking 2-cycle $c$ in $\hat{W}$. However note that $c$ intersects $E$ along a 1-cycle $\gamma$. 
To see the cycle $c$ and the fact that its volume vanishes if and only if the 1-cycle $\gamma$ shrinks, consider the local situation in $W_{1}$ described by choosing

$$
f=f_{0}+\rho f_{1}=\left(-3 a^{2}\right)+\rho f_{1}, \quad g=g_{0}+\rho g_{1}=\left(2 a^{3}-\epsilon\right)+\rho g_{1},
$$

in (3.1). For small $\epsilon$, the heterotic torus at $\rho=0$ has a small $S^{1}$ described by

$$
\gamma: y^{2}+x^{2}=\epsilon
$$

Away from $\rho=0$ there is still an $S^{1}, \gamma(\rho)$, with a radius $\sqrt{\epsilon(\rho)}$ depending on the value of $\rho$. From the vanishing of the discriminant $\Delta=\Delta_{0}+\rho \Delta_{1}$ we see that $\gamma(\rho)$ shrinks to zero size at a point $\rho_{0}=-\Delta_{0} / \Delta_{1}$. We can therefore define a two 2-chain $K^{(1)}$ in $W_{1}$ from gluing together 1-cycles $\gamma(\rho)$ along the interval $\rho=\left[0, \rho_{0}\right]$.

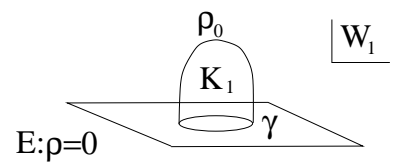

Fig. 2: The 2-chain $K^{(1)}$ in $W_{1}$.

Note that the cycle $\gamma=\gamma(0)$ in $E$ shrinks iff $\Delta_{0}=0$, which implies $\rho_{0} \rightarrow 0$. Thus precisely if $\gamma$ shrinks, $K^{(1)}$ shrinks, too. In fact the volume of the 2 -chain $K^{(1)}$ is determined by the integral

$$
\int_{K^{(1)}} \Omega \sim \int_{K^{(1)}} \frac{d x d \rho}{y}=\frac{\pi \epsilon}{\sqrt{3 a}\left(a f_{1}+g_{1}\right)},
$$

where the proportionality constant depends on the normalization of the 2 -form $\Omega$. In the total manifold $\hat{W}$ we obtain a vanishing 2 -cycle from the two vanishing 2 chains $K^{(\alpha)} \subset W_{\alpha}$ joining along the vanishing cycle $\gamma$. Note that we can write the discriminant of $\hat{W}$ as $\Delta(\hat{W})=\Delta\left(W_{1}\right) \cdot \Delta\left(W_{2}\right)$, which vanishes to second order at $\mu=\rho=0$ due to the first order vanishing of $\Delta\left(W_{i}\right)$.

Thus this second kind of degeneration is related to a geometric degeneration of the heterotic torus $E$. This does not make too much sense in the case of the torus, since its degenerations are very limited and in fact at infinite distance in the moduli spacel 1 . We can also combine the two kind of degenerations and bring together several singular fibers from

1 However note that in this limit we have $U=\infty=T$ so we can interpreted the $S U(2)$ gauge symmetry of the type IIA string on the vanishing 2-cycle as the heterotic gauge symmetry enhancement at $T=U$ in the limit $T \rightarrow \infty$. 
$W_{1}$ and $W_{2}$ at the intersection. From the above this should correspond to a degeneration of the bundle on top of the singular heterotic geometry.

Having discussed this toy example, let us consider the case of heterotic string on a non-trivial Calabi-Yau $Z_{n}, n>1$, in particular for $Z=K 3$. The new ingredient is that we can now have geometric singularities of $Z$ at finite distance in the moduli, in particular ADE singularities of ALE spaces. Let us fiber the eight-dimensional picture in Fig. 1 over an extra $\mathbf{P}^{1}$, parametrized by $t$. The local structure of the 3 -fold geometry $\hat{W}$ for small 2-cycles $c_{i}$ in $Z$ is similar as in eq.(4.1) with an extra dependence on the base $\mathbf{P}^{1}$ :

$$
f=f_{0}(t)+\rho f_{1}(t), \quad g=g_{0}(t)+\rho g_{1}(t) .
$$

Similarly we can split the discriminant of the elliptic fibration as $\Delta=\Delta_{0}(t)+\rho \Delta_{1}(t)$. Near a $G$ singularity of the K3, the zeros of $\Delta_{0}$ are described by

$$
\Delta_{0}=\prod_{i}^{\delta}\left(t-t_{i}\left(a_{k}\right)\right)=t^{\delta}+\ldots
$$

where $a_{k}, k=1, \ldots, \mathrm{rk} G$ denote the deformations of the singularity and $\delta$ is a certain integer that counts the number of simple singular fibers needed to produce the $G$ singularity in the elliptic fibration2. Classically, the $G$ singularity is recovered in the limit, where the perturbations $a_{k}$ become zero.

Moving away from $\rho=0$ to describe the 3 -fold $\hat{W}$, the zeros $t_{i}$ of the discriminant $\Delta$ depend on $\rho$ :

$$
\Delta=\Delta_{0}+\rho \Delta_{1}=\prod_{i}^{\delta}\left(t-t_{i}\left(a_{k}, \rho\right)\right) .
$$

Note that for each pair of $t_{i}\left(a_{k}, \rho\right)$, we can define a 2-cycle $c_{i j}(\rho)$ by stringing together 1 cycles in $E$ along the interval $\left[t_{i}\left(a_{k}, \rho\right), t_{j}\left(a_{k}, \rho\right)\right]$, very similar as we did in the construction of the 2-chains $K^{(\alpha)}$. Moreover the 2-cycle $c_{i j}(\rho)$ attaches to a 2-cycle $c_{i j}$ of the K3 $Z$ for $\rho=0$. In addition it shrinks to zero size at a point $\rho=\rho_{i j}$ where

$$
t_{i}\left(a_{k}, \rho_{i j}\right)=t_{j}\left(a_{k}, \rho_{i j}\right)
$$

Very similar as in the previous case we can now build 3 -chains $K_{i j}^{(\alpha)}$ by gluing together the 2-spheres $c_{i j}(\rho)$ along the interval $\left[0, \rho_{i j}\right]$.

2 E.g. $\delta=N$ for $S U(N)$ and more generally $\delta=\operatorname{rk} G+1+\nu$, where $\nu$ is the number of trivalent vertices in the Dynkin diagram of $G$. See ref. 14. 
Finally note that for very similar reasons as for the 2-chains constructed before, the volume of the 3 -chain $K_{i j}^{(\alpha)}$ vanishes precisely if the 2-cycle $c_{i j}$ shrinks and in particular all 3-chains shrink near the singularity of the heterotic K3 $Z$

$$
a_{k} \rightarrow 0 \Rightarrow \rho_{i j} \rightarrow 0
$$

One can calculate also the volume of the individual 3-chains $K_{i j}^{(\alpha)}$ similarly as in (4.2).

We have therefore succeeded to establish a 1-1 correspondence between the small 2-cycles $c_{i j}$ of the resolved ADE singularity and small 3-cycles $C_{i j}=K_{i j}^{(1)} \cup K_{i j}^{(2)}$ of a singularity reached in the complex structure of the type II compactification manifold $\hat{W}$. The lattice $H_{3}(\hat{W})$ inherits the ADE structure from the lattice $H_{2}(Z)$. Note that the cycles $C_{i j}$ intersect each other according to the ADE Dynkin diagram. This fits with the physics expectations that the instanton corrections to the three-dimensional gauge theory represented by type IIB on $\hat{W}$ arise from the monopoles and dyons, as opposed to the wrappings of purely electric cycles.

The singularity of the type II manifold $\hat{W}$ is precisely the one which is known to produce the $G$ gauge theory in three dimensions in a type IIB compactification on $\hat{W} \times S^{1}$ [9]. Note that we needed the extra $S^{1}$ to translate from the heterotic type IIA dual that describes the "Higgs branch" of hypermultiplets, to the Coulomb branch of the $G$ gauge theory of the type IIB field theory limit, which corresponds to the dual theory in the sense of [5], [6]. Let us also identify the states dual to the monopoles from D3 branes wrapped on $C_{i j}$. In the T-dual type IIA theory they become D4 branes wrapped on $C_{i j} \times S^{1}$. In the heterotic theory they become 5-branes wrapped on $c_{i j} \times T^{3}$.

Let us consider now possible generalizations of the above picture by moving additional singular elliptic fibers into the heterotic singularity. As discussed in the eight-dimensional picture, if we let collide fibers at some point $\rho=\rho^{\prime}$ of the base of the elliptic surface $W_{i}$, this corresponds to a degeneration of the heterotic bundle. Moving this point into the ADE singularity, $\rho^{\prime} \rightarrow 0$, we expect that in general the gauge bundle is not trivial on the singularity. This would correspond to theories with non-perturbative heterotic dynamics, such as the extra gauge symmetry enhancement discussed in [10].

Let us first consider a simpler case, where it is easier to find the answer. In the sixdimensional compactification we can also consider moving in additional singular elliptic fibers into the singularity using the $t$ direction. In this way we can keep the singularity of the elliptic surface $W_{i}$, and thus the bundle, fixed on the singularity. In particular, if we choose a totally trivial gauge bundle there are 24 small instantons located at points $t=t_{i}$ of the base of $\mathrm{Z}$. The case where some of these positions coincide with the location 
of the ADE singularity has been studied in great detail in [15] [13]. With some effort one can show that after moving in such points there are new 3-cycles in $\hat{W}$ that correspond to adding matter in the gauge theory following the rules of geometric engineering of type II strings. Note that we can only get matter that can be obtained from adjoint breaking of a higher rank gauge group [16] in this way. We will take a shortcut here that uses the results on three-dimensional mirror symmetry described in [6] [7], which will lead us also to a natural conjecture for new three-dimensional dualities. Specifically, ref. [0] established a series of three-dimensional dualities using geometric engineering of type II on geometric singularities, which is precisely the situation that we consider. It was shown that if the type IIB theory on a Calabi-Yau $W$ describes a $U(N)$ theory with $N_{f}=k$ fundamentals, than the type IIA theory on the same manifold describes the Higgs branch of a dual theory with gauge group $U(1) \cdot U(2) \cdots U(N-1) \cdot U(N)^{k-2 N+1} \cdot U(N-1) \cdots U(2) \cdot U(1)$. On the other hand [15] [13] this is precisely the gauge theory of $k$ small instantons on a $S U(N)$ singularity of K3! Thus we see that by considering the field theory limit of the heterotic theory with small instantons on the K3 singularity it essentially follows from the results in [7] that the heterotic moduli of the $A_{N-1}$ singularity with $k$ instantons is the same as that of the three-dimensional $S U(N)$ theory with $N_{f}=k$.

It is natural to wonder whether this relation is true more generally, also in those cases, where three-dimensional dualities have not yet been established or even formulated. The prediction would be that the theory dual to the three-dimensional gauge theory with gauge group $G$ and $k$ matter multiplets is the gauge theory with gauge group $\tilde{G}$, where $\tilde{G}$ is the extra local gauge group that appears if $k$ small heterotic instantons collide with a $G$ singularity of K3.

Let us now come back to the case where we move singular fibers of the elliptic surface $W_{1}$ into the ADE singularity. We have in the moment no good understanding of what the three-dimensional theories associated to such a degeneration may be, however there is also a plausible candidate for this case from the geometric engineering of type II strings. Note first that if we take the base to be $\mathbf{P}^{1} \times \mathbf{P}^{1}$, we can interpret any of the two $\mathbf{P}^{1}$ 's as the base of the heterotic K3. Accordingly we have two heterotic theories that are related by

3 It is worth noticing that this result is consistent with a proposal for mirror symmetry of F-theory formulated in [17] and derived and refined in [10]. In particular it states that if F-theory on a 3 -fold $\tilde{W}$ describes the Coulomb branch of $k$ small heterotic instantons on a $G$ singularity of K3, than the mirror $\tilde{W}^{*}$ is dual to a heterotic theory on the Higgs branch describing a large $G$ instanton with instanton number $k$. After a $T^{3}$ compactification, the two theories are related by the three-dimensional duality as above. 
heterotic-heterotic duality [18]. An extension of this duality for singular K3's and bundle singularities exists and has been described in [10]. In the previous case we have considered having a $G$ singularity in the $\mathrm{K} 3$ with base $\mathbf{P}^{1}$ parametrized by $t$. Of course nothing changes if we consider instead only a singularity $G^{\prime}$ in the heterotic theory with base the other $\mathbf{P}^{1}(s, \tilde{s})$. If we now consider a manifold where the $G$ and $G^{\prime}$ singularities collide, we have in some sense a collision of two gauge theories with groups $G$ and $G^{\prime}$. This reminds very much of the structure of the conformal field theories engineered from type II strings in [19]. In this case one has a gauge group $G=\prod S U\left(k n_{i}\right)$, with $n_{i}$ the Dynkin indices of a gauge group $G^{\prime}$ and bi-fundamentals in each group factors connected by a link in the Dynkin diagram. The coupling constants of the gauge theory are in fact related to the Coulomb parameter of a $G^{\prime}$ gauge theory which is however broken to a trivial $U(1)^{\mathrm{rk} G^{\prime}}$ factor in the region where we observe the $G$ gauge theory. However it is also possible to to move towards the origin of the Coulomb branch of $G$ and $G^{\prime}$ at the same time. One obtains in this limit an exotic gravity free theory with string like degrees of freedom [19]. The compactification of this theory on $S^{1}$ seems to be a good candidate for the threedimensional theories related to a collision of a singularity in $W_{1}$ with an ADE singularity in $Z$.

\section{Heterotic CFT on Calabi-Yau 3-fold singularities and Kazama-Suzuki mod- els in two dimensions}

By the same arguments as in the previous section we can construct more generally a correspondence between $m+1$-chains in the stable degeneration of a Calabi-Yau $m+1$ fold $W_{m+1}$ and $m$ cycles in the intersection of its two components which is an elliptic $m$-dimensional Calabi-Yau manifold. For the case $m=3$ we obtain a correspondence between the heterotic CFT on the isolated singularity

$$
y^{2}+H(x, s, t)=0,
$$

and type II strings on a Calabi-Yau 4-fold with a corresponding singularity of one dimension higher described locally by a similar equation with $H$ replaced by $H+w^{2}$. Here $H=0$ describes the ADE surface singularities

$$
\begin{array}{llll}
H=s^{n}+t^{2}+x^{2} & A_{n-1}, & H=s^{3}+s t^{3}+x^{2} & E_{7}, \\
H=s^{n}+s t^{2}+x^{2} & D_{n+1}, & H=s^{3}+t^{5}+x^{2} & E_{8} . \\
H=s^{3}+t^{4}+x^{2} & E_{6}, & &
\end{array}
$$


The field theory limit of type IIA theory on these singularities has been identified in [20] as a Kazama-Suzuki model based on a coset that depends on the choice of a RR background on 4-cycles. The RR fields of type IIA map to gauge fields of the hetrotic string. We conclude that the heterotic CFT on the 3-fold singularity with appropriate gauge field background is on the same moduli as the corresponding Kazama-Suzuki model. A simple consistency check on this equivalence is the following one. Using linear sigma models it was argued in [1] that the heterotic CFT should be smooth on a singular Calabi-Yau $n$-fold for any $n$ as long as the gauge fields are trivial on the singularity. For trivial RRfields one obtains a massive Kazama-Suzuki model with no light degrees of freedom. This matches the non-singular behavior of the heterotic theory advocated in [1]. It would be interesting to compare in detail the moduli spaces for the more interesting case with nontrivial background fields, in particular when the heterotic string is compactified on the $(2,2)$ theory.

\section{Discussion}

The equivalence of the moduli space of the heterotic CFT on ADE singularities and the moduli space of three dimensional gauge theories, or the dual monopole moduli spaces, is an interesting tool to study several aspects. E.g., the metric of the Atiyah-Hitchin manifold is known [2] and one can extract the world-sheet instanton expansion of the heterotic CFT, similarly as one obtains the world-sheet instantons of the type II string from mirror symmetry. By an appropriate reparametrization, we expect to reproduce the D2 instanton expansion of the type IIA string on $\hat{W}$. This allows to analyze a new class of brane wrappings. It is interesting to note in this context that it is easy to verify that the Atiyah-Hitchin metric is compatible with the claim in [3] that in the gauge theory variables there is only a single instanton contribution; this is a first non-trivial compatibility check. Reversing the logic it would also be very interesting to study metrics on monopole moduli spaces from the CFT approach. Similarly it would be interesting to study the relation between instanton corrections to the metric of the heterotic 3-fold and the two-dimensional Kazama-Suzuki models.

Note added: While preparing this note for publication, two related papers appeared on the subject. Ref.[21] gives an independent derivation of the conjecture of ref.[1] using Mtheory. Ref. [22] treats the special case $G=A_{1}$ case, already analyzed in [1], using a "new" variant of geometric engineering for heterotic strings. In fact this is the same geometric 
engineering used in this papert and derived and studied in detail by local mirror symmetry in 10$]$ 19]. 23.

\section{Acknowledgments:}

We would like to thank W. Lerche and Y. Oz for valuable discussions.

4 In particular the definition of the geometric limit on p.6 in 22] is a special example of the analysis in App. A of [10]. 


\section{References}

[1] E. Witten, Heterotic string conformal field theory and $A-D$-E singularities, hepth/9909229.

[2] M.F. Atiyah and N. Hitchin, The geometry and dynamics of magnetic monopoles (Princeton University Press, 1988),

[3] N. Seiberg and E. Witten, Gauge dynamics and compactification to three dimensions, hep-th/9607163.

[4] A. Sen, Adv. Theor. Math. Phys. 1 (1998) 115.

[5] K. Intriligator and N. Seiberg, Phys. Lett. $\underline{B 387}$ (1996) 513.

[6] J. de Boer, K. Hori, H. Ooguri and Y. Oz, Nucl. Phys. B493 (1997) 101;

M. Porrati and A. Zaffaroni, Nucl. Phys. B490 (1997) 107.

[7] K. Hori, H. Ooguri and C. Vafa, Nucl. Phys. B504 (1997) 147.

[8] E. Witten, Nucl. Phys. B4443 (1995) 85.

[9] A. Klemm, W. Lerche, P. Mayr, C. Vafa, N. Warner, Nucl. Phys. B477 (1996) 746;

S. Katz, A. Klemm and C. Vafa, Nucl. Phys. B497 (1997) 173;

S. Katz, P. Mayr and C. Vafa, Adv. Theor. Math. Phys. 1 (1998) 53.

[10] P. Berglund and P. Mayr, Adv. Theor. Math. Phys. $\underline{2}$ (1999) 1307.

[11] D.R. Morrison and C. Vafa, Nucl. Phys. $\underline{B 476}$ (1996) 437; Nucl. Phys. $\underline{B 473}$ (1996) 74.

[12] R. Friedman, J. Morgan and E. Witten, Comm. Math. Phys. 187 (1997) 679.

[13] P.S. Aspinwall and D.R. Morrison, Nucl. Phys. B503 (1997) 533.

[14] M. Bershadsky et al., Nucl. Phys. $\underline{B 481}$ (1996) 215.

[15] K. Intriligator, Nucl. Phys. $\underline{B 496}$ (1997) 177;

J. Blum and K. Intriligator, Nucl. Phys. B506 (1997) 199, Nucl. Phys. B B506 (1997) 223.

[16] S. Katz and C. Vafa, Nucl. Phys. $\underline{B 497}$ (1997) 146.

[17] E. Perevalov and G. Rajesh, Phys. Rev. Lett. $\underline{79}$ (1997) 2931.

[18] M.J. Duff, R. Minasian and E. Witten, Nucl. Phys. B465 (1996) 413;

D.R. Morrison and C. Vafa, Nucl. Phys. $\underline{B 476}$ (1996) 437; Nucl. Phys. $\underline{B 473}$ (1996) 74 .

[19] S. Katz, P. Mayr and C. Vafa, Adv. Theor. Math. Phys. 1 (1998) 53.

[20] S. Gukov, C. Vafa and E. Witten, CFT's from Calabi-Yau four-folds, hep-th/9906070. 
[21] M. Rozali, Hypermultiplet moduli space and three dimensional gauge theories, hepth/9910238.

[22] R.S. Aspinwall and R. Plesser, Heterotic string corrections from the dual type II string, hep-th 9910248 .

[23] For an introduction and background material see P. Mayr, hep-th 9904115; hepth/9910216; D-branes and F-theory, lectures the Trieste Spring School 1999, to appear in the proceedings. 